\title{
Gender differences in spatial navigation: Characterizing wayfinding behaviors
}

\author{
Ascher K. Munion ${ }^{1}$ • Jeanine K. Stefanucci ${ }^{1}$ - Ericka Rovira ${ }^{2} \cdot$ Peter Squire $^{3}$ - Michael Hendricks ${ }^{4}$
}

Published online: 20 August 2019

(C) The Psychonomic Society, Inc. 2019

\begin{abstract}
Men show a consistent spatial navigation advantage over women, which is often attributed to their increased use of survey spatial strategies. But what about men's navigation gives them an advantage? One possibility is that the way in which men explore environments is fundamentally different, leading to better navigational performance. To test this possibility, this study investigated whether there are gender differences in wayfinding behaviors during navigation that relate to navigational success in a realworld, large-scale, unconstrained navigation task. West Point cadets were given a masked GPS tracker and sent into a large-scale, natural environment to locate targets indicated on maps. We assessed how they explored the environment by computing three measures from the GPS tracks and related these measures to their ability to find the assigned target locations. We also tested whether their self-reported spatial ability related to navigational success. Results showed that males performed better than females, which replicates prior work. Further, traveling longer distances without changing course, pausing less, and fewer returns to previously visited locations were significantly related to the ability to locate the correct target. Consistent with full mediation, the significant relationship between gender and navigational success is fully accounted for by men and women producing different wayfinding behaviors, which in turn predict differences in navigational success. Further, there was no unique relationship between self-reported spatial skills and navigational success. This study is a first step toward showing the relationship between gender, wayfinding behaviors, and navigational success in a natural, real-world navigation task.
\end{abstract}

Keywords Navigation · Gender differences · Wayfinding $\cdot$ Spatial cognition

Navigation and wayfinding are daily parts of human experience, whether it is driving to the grocery store or hiking up a mountain. New research suggests that men may actually be more efficient at navigating than women (Boone, Gong, \& Hegarty, 2018), as assessed through both selfreport and virtual navigation tasks. When men and women freely explore environments (virtual or real), many studies find male advantages in navigation performance (Coluccia $\&$ Louse, 2004). Further, exposure to a map prior to

Ascher K. Munion

u1008334@utah.edu

1 Department of Psychology, University of Utah, 380 S 1530 E, Room 502, Salt Lake City, UT 84112, USA

2 Department of Psychology, United States Military Academy West Point, West Point, NY, USA

3 Office of Naval Research, Arlington, VA, USA

4 Center for Environmental and Geographic Sciences, United States Military Academy West Point, West Point, NY, USA performing a wayfinding task, and interactive use of maps for navigation during the task, does not eliminate the gender differences in wayfinding success (Castelli, LatiniCorazzini, \& Geminiani, 2008; Coluccia, \& Louse, 2004; Malinowski, 2001; Malinowski \& Gillespie, 2001). Men perform better than women on active wayfinding tasks when they have access to maps, even when accounting for familiarity with map use. Further, navigational success, as defined by both speed and accuracy, is higher when navigating based on cardinal directions compared with landmark directions in virtual environments (Hund \& Minarik, 2006), but women tend to navigate based on route strategies more so than with cardinal directions (Fields \& Shelton, 2006). However, when men and women are both primed to navigate with landmarks, the gender differences shrink (Hund \& Minarik, 2006), suggesting that priming for cue use can influence navigational outcomes.

However, little work has assessed exploration in real environments. Malinowski and Gillespie (2001) assessed sex differences in a real-world wayfinding task, in which participants used maps and compasses to navigate a large-scale wooded 
area. They found that men performed better on a small-scale spatial task (mental rotation), and that this superior performance was highly correlated with a real-world, large-scale orienteering task. However, the difference in men and women's performance on the wayfinding task was not significant, so it is unclear if the way in which people explored-or moved through the environment-affected the results in that study. Montello, Lovelace, Golledge, and Self (1999) showed that males could better estimate route distances and straightline direction of the start (see also Silverman et al., 2000) and finish of a route when spatial knowledge was acquired by navigating a new route through a real-world campus environment. Further, Ishikawa and Montello (2006) investigated individual differences in acquisition of spatial knowledge when learning paths through a real environment for 10 consecutive weeks. Surprisingly, participants were either generally good or bad, but on average did not show much improvement in spatial knowledge at the tenth exposure to the learned environment compared with the first, suggesting that participants either acquired spatial knowledge quickly or they did not acquire much at all over time.

Another measure of individual differences in spatial ability - the Santa Barbara Sense of Direction (SBSOD) - is a self-report measure that correlates with spatial knowledge (Hegarty, Richardson, Montello, Lovelace, \& Subbiah, 2002) and is frequently used as a proxy for navigational ability. The SBSOD is correlated with wayfinding abilities in virtual tasks, with a stronger relationship for immersive virtual reality as compared with video presentation of stimuli (Hegarty, Montello, Richardson, Ishikawa, \& Lovelace, 2006). However, the link between this self-report measure and individual differences in cognate representations of space, both in virtual and real-world environments, is not always consistent (Weisberg, Schinazi, Newcombe, Shipley, \& Epstein, 2014). Ploran, Rovira, Thompson, and Parasuraman (2015) found that when considering both SBSOD and spatial working memory, only spatial working memory predicted navigational success in a large-scale wayfinding task. Similarly, Rovira et al. (2016) showed the same samples' sense of direction (measured by both SBSOD and a brain-derived neurotrophic factor) was not uniquely associated with navigational success, suggesting self-report of navigational ability may not be the best predictor of wayfinding skills.

Because self-reports frequently do not capture the nuance of navigational ability, virtual environments and map-based laboratory tasks are often used to assess navigational ability (Castelli et al., 2008). It is still unclear whether virtual environments approximate real-world exploration (Coluccia \& Louse, 2004). Two constraints in virtual environment navigation are the size of the virtual environments and the speed and mechanisms by which participants move through them (frequently, participants view a video of a path rather than navigating freely;
Fields \& Shelton, 2006; Sandstrom, Kaufman, \& Huettel, 1998; Weisberg et al. 2014). Two prior studies in a virtual environment allowed for free exploration and found that women looking for objects in a large virtual environment $(\sim 1 \mathrm{~km}$ in diameter) produced different exploration patterns than men (Gagnon, Cashdan, Stefanucci, \& Creem-Regehr, 2016; Gagnon et al., 2018). These different patterns reflected women exploring more "cautiously," with more revisited locations and pauses compared with men. Further, the increase in pausing and revisiting reduced navigational performance as assessed through navigating back to found items and pointing to targets after exploration ceased. Gagnon et al. (2018) replicated the finding that females revisited more locations during a free exploration task but also found that males tended to exhibit exploration patterns that allowed them to spread through the environment more quickly. These observed exploration patterns partially accounted for gender differences in the ability to efficiently return to or point to locations within the environment, providing preliminary evidence that how people explore environments predicts future navigational success. This claim has not been tested with a real-world exploration task.

In the current study, we investigated whether participants' self-reported spatial abilities (SBSOD) gender related to how they explored a large-scale, unconstrained, real-world environment, and their ability to correctly locate targets. We hypothesized (based on the findings from Gagnon et al., 2016, and Gagnon et al., 2018, in desktop virtual environments) that gender differences in real-world wayfinding behaviors with male and female cadets at West Point would relate to navigational success. Specifically, we expected that the ways in which men and women moved throughout the environment would be different, and that these differences in wayfinding behaviors would predict participants' success in correctly locating targets. Using the SBSOD, we also examined if realworld behavioral performance uniquely related to selfreported navigation ability.

\section{Method}

\section{Participants}

A total of 518 cadets ( 89 women, 429 men), in the second year of their West Point program, completed a required large-scale navigation task to assess their wayfinding skills. A total of 186 participants completed various self-reported navigational skills and strategies assessments, from which we used the Santa Barbara Sense of Direction (SBSOD; $\alpha=.706$ ) questionnaire (Hegarty et al., 2002). The cadets who completed the navigational strategies assessment were $66.5 \%$ (124) men (coded 1) and 33.5\% (69) women (coded 0). 


\section{Procedure}

In a large-scale wayfinding task, cadets located flags in rugged, wooded terrain (the same area used in Malinowski \& Gillespie, 2001). Participants were given a day of training to familiarize them with the task prior to data collection, and all participants demonstrated competency with the task and the map and compass use prior to data collection. Participants navigated to five sets of latitude/longitude coordinates of flags, using a map and compass, while a screen-less GPS tracker sampled once a second for the entirety of the wayfinding task (on average, 2.37 hours).

\section{Target locating (navigational success)}

Participants were told to locate flag locations in the wooded environment using only their map and compass. There were 30 possible flag locations, each a quarter mile or more apart, with clusters of three flags that were approximately 50-100 feet apart at each location. Distance and difficulty of the terrain to reach the flags were roughly equivalent across participants. No more than three participants received the same set, in order to lower the likelihood of coordination of search patterns to aid in performance. Each course (with two exceptions) was assigned to at least one male and one female cadet, to control for differences in task difficulty across genders.

Participants marked scorecards to indicate which flag they had found. These indicators gave two levels of assessment of performance - the general ability to identify the correct flag in the correct cluster (1 point), a correct cluster but not correct flag ( 0.5 point), or neither ( 0 points), according to the coordinates given to the participant.

\section{Measures of wayfinding behaviors}

The GPS tracks were analyzed to provide different conceptualizations of how the cadets moved through the space, which taken together provides a cohesive description of cadets' wayfinding behaviors. The development of the measures used herein (Gagnon et al., 2016; Gagnon et al., 2018) was motivated by extensive research in behavioral ecology devoted to the study and quantification of animal movement and how it relates to achieving adaptive goals like foraging for food and finding mates (Turchin, 1998). How people explore virtual environments and perform in virtual navigation tasks was well described by measures of directional persistence, pausing, and revisiting (Gagnon et al., 2016; Gagnon et al., 2018). These measures allow us to understand how dynamic wayfinding behaviors may relate to navigational success. We expect that the way in which people explore environments will be related to their navigational performance such that males exhibit more efficient and confident wayfinding behaviors (less revisiting and pausing), and these more effective wayfinding behaviors will mediate the oft-observed gender differences in navigational success. Using specialty scripts written in R, these separate measures were computed for the GPS tracks produced by the cadets.

\section{Directional persistence}

Directional persistence offers a way to extract relative distance information from a spatial layout, on a scale proportional to the length of the directional persistence. Directional persistence was calculated by multiplying the individual's speed (change in distance over change in time) by the cosine of their turning angle. Therefore, traveling at maximum speed in a straight line would yield a directional persistence estimate of the participants' maximum speed. Traveling at maximum speed, but making a $90^{\circ}$ turn, would yield a directional persistence estimate of zero. Directional persistence was calculated over 1-second intervals for the entirety of each cadet's navigation, then averaged to create an overall directional persistence score for each participant. We argue that directional persistence (as measured here) can, in part, provide an index of confidence in navigation. Further, as with the analogous measure (diffusion) in Gagnon et al. (2018), directional persistence gives spatial information about objective patterns of behavior that, along with the following two measures, may represent caution in wayfinding as compared with selfreports of caution.

\section{Pausing}

Pausing was calculated by dividing the total number of seconds spent not moving by the total time spent navigating or exploring during the task. A pause was defined as consecutive samples with the same $\mathrm{X}, \mathrm{Y}$, and $\mathrm{Z}$ values, and the number of consecutive samples with the same position values was used to infer the number of seconds for a given pause. The lengths (in seconds) of the pauses were summed and divided by the total number of seconds in the wayfinding trajectory.

\section{Revisiting}

To calculate cadets' revisiting behaviors during navigation, we computed a 45 - $\mathrm{ft}$ radius around each location along a participant's trajectory (see Gagnon et al., 2018, for use of this measure in virtual navigation). The decision of 45 feet was based on the distance between flags at locations, the rate of movement throughout the task, and the visual density of the environment cadets were exploring. For each position in the participant's trajectory, the euclidean distance between the current position and all other previous positions was calculated, and all positions with a euclidean distance equal to or less than $45 \mathrm{ft}$ were identified. Revisiting positions were defined as any of the positions within the 45 - $\mathrm{ft}$ radius in which the 
participant left and then reentered that area. Finally, the average number of revisiting positions across all samples in the wayfinding trajectory was computed, indicating the overall amount of revisiting behavior

\section{Analysis strategy}

The goals of this study are to (1) categorize how participants moved in a real-world environment during a largescale navigation task, and (2) to determine whether the characterizations of movement patterns (directional persistence, revisiting, and pausing) relate to navigational success in the task. Using structural equations modeling (path diagram shown in Fig. 1), the wayfinding behaviors (pausing, revisiting, and directional persistence) were loaded onto a latent variable, conceptualized as a comprehensive measure of wayfinding behaviors, which was predicted by gender and predicted navigational success, consistent with mediation. We expected that men would produce on average more directional persistence, less pausing, and less revisiting than women, which in turn would account for the male advantage on navigational performance (accurately locating targets). SBSOD was correlated with gender and predicted ability to accurately locate the target, testing if self-reported navigational ability uniquely predicted target location. Self-report of comfort with navigational aids was included as a covariate, to control for potential gender and individual differences in comfort with navigational tool use. The proposed model tests the connections between self-report of navigational ability (SBSOD) and ability to locate a target, while providing behavioral quantifications of the proposed differences in how men and women move through space. Importantly, although there is a theoretical connection between production of different wayfinding behaviors and navigational success, there is no linear dependency between these measures. Participants could theoretically pause more, revisit more, and have less directional persistence, but still find the correct flag, and thus get full points for navigational success. Thus, we are testing empirically whether how cadets move throughout the environment (wayfind) is related to locating the correct target (navigational success).

Models were run in Mplus 7.31 using a maximum likelihood estimator. Model fit was assessed using CFI (>.90) and SRMR $(<0.08$ indicating good fit; Hu \& Bentler, 1999). Missingness ranged from 9\%- to $12.5 \%, 10$ multiply imputed data files accounted for this relatively low missingness (see Graham, 2009). A significant bootstrapped indirect effect $(10,000$ samples) tested mediational plausibility (for meditation details, see Kenny, 2008). The data and R scripts for the experiments reported here are available by request, and the experiment was not preregistered.

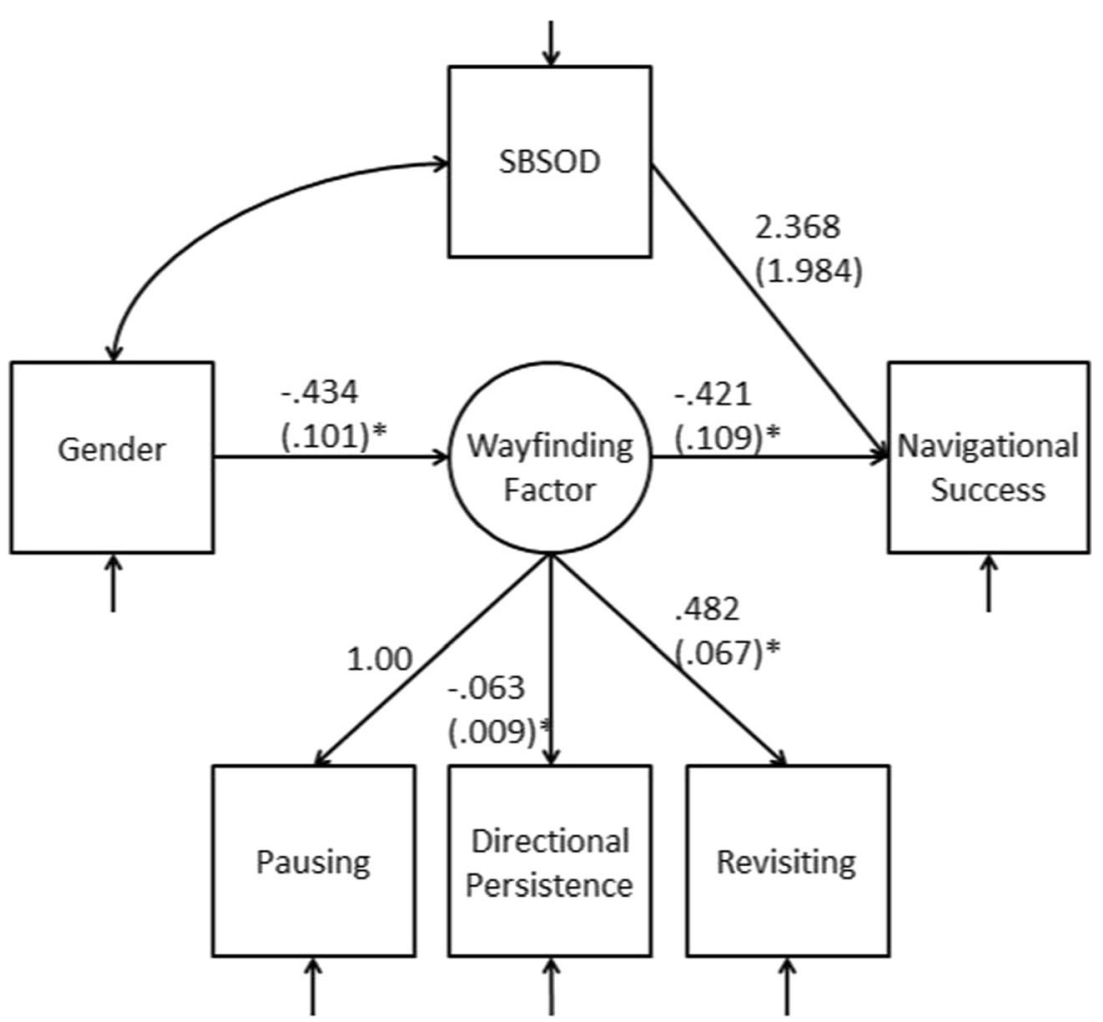

Fig. 1 Full mediation model. Standard errors are in parenthesis. The nonsignificant path (direct effect from gender to navigational success) and the covariate of comfort with navigational aids are not represented for simplicity of path diagram 
Table 1 Correlation table for all variables of interest

\begin{tabular}{lllllll}
\hline & Gender & SBSOD & Revisiting & Pausing & Directional persistence & Navigational success \\
\hline Gender & 1.00 & $.078^{*}$ & $-.134^{*}$ & $-.111^{*}$ & $.134^{*}$ & $.217^{*}$ \\
SBSOD & - & 1.00 & $.206^{*}$ & $-.154^{*}$ & $.205^{*}$ & .130 \\
Revisiting & - & - & 1.00 & $.546^{* *}$ & $-.366^{* *}$ & $-.475^{* *}$ \\
Pausing & - & - & - & 1.00 & $-.205^{* *}$ & $-.573^{* *}$ \\
Directional persistence & - & - & - & - & 1.00 & $.563^{* *}$ \\
Navigational success & - & - & - & - & - & 1.00 \\
\hline
\end{tabular}

$*=$ significance at the .05 level. $* *=$ significance at the .01 level

\section{Results}

Correlations between all variables of interest are presented in Table 1. Figure 1 shows the mediation model, and presents all estimated coefficients and $R^{2}$ values. ${ }^{1}$ This model was of adequate fit, as assessed by a combination of chi-square test of model fit, $\chi(7)=8.590, p=.285, \mathrm{CFI}=.996, \mathrm{SRMR}=.024$. The three measures of wayfinding behaviors maintained the expected loading directions in relation to each other. Gender predicted the wayfinding behaviors factor (with higher values indicating more caution), which in turn predicted the ability to locate the target (flag), such that males performed fewer pausing and revisiting behaviors and showed more directional persistence, thereby locating more correct flags. Gender differences in finding the correct flag were fully accounted for by the indirect path. This finding is consistent with mediation with gender significantly predicting wayfinding behaviors, which predicted navigational success. The significant indirect effect $(b=.184,95 \%$ CI $[.101, .263])$ implies the relationship between gender and navigational success is fully described by men and women moving throughout the environment differently. Finally, self-report of navigational ability (SBSOD) was not significantly related to identifying the correct cluster and flag in the full path model (Table 2).

\section{Discussion}

Measures previously used to assess relationships between virtual exploration and navigation also capture real-world wayfinding behaviors' relationship to cadets' ability to correctly locate targets during navigation of a large-scale, unconstrained space. Consistent with our hypothesis (motivated by virtual reality work by Gagnon et al., 2016; Gagnon et al., 2018), cadets with higher degrees of pausing and revisiting and lower degrees of directional persistence were significantly worse at identifying the correct flag. Men performed better, on average, than women. We were curious to see if the female cadets would have wayfinding behaviors or self-reported

\footnotetext{
${ }^{1}$ Results of the nested, nonmediation model is provided in the Fig. 2.
}

spatial skills comparable with male cadets, given that they self-selected into a career requiring high use of navigational and spatial skills. However, our sample showed the laypopulation effects of an overall male advantage in navigational performance, and higher self-reports of spatial abilities for males as compared with females.

We hypothesized that the relationship between gender and navigational success would be mediated or fully described by differences in wayfinding behaviors produced during the task. That is, we believed that the differences in performance between men and women would be fully or partially accounted for by their wayfinding behavior. We propose this wayfinding behavior taxonomy as a meaningful description of how the commonly found gender differences in spatial cognition and navigation play out behaviorally. Our results were consistent with male and female cadets' production of different wayfinding behaviors accounting for the relationship between gender and locating the correct flag. Thus, men's better navigational performance was accounted for by more directional persistence and less revisiting and pausing during the task compared with women's. These findings are consistent with how someone explores predicting their final ability to locate the correct target. Further, men and women produced different wayfinding behaviors during exploration of this large, wooded, real-world environment. The gender difference in wayfinding behaviors fully accounted for the relationship between gender and navigational success, suggesting that gender does not predict differences in ability to locate the correct target over and above how people are wayfinding. We provide this meditational model as a data-reduction argumentcharacterizing the gender differences in wayfinding - rather than a strong causal argument. That is, the relationship between gender and navigational success is fully explained by the indirect paths through the wayfinding behaviors; however, gender may not be the only or most proximal cause for this relationship.

Further, we found no relationship between SBSOD scores and navigational success while accounting for wayfinding behaviors. The SBSOD is frequently used in small-scale navigation tasks and may not be a powerful predictor of navigational success in this task, or may have high collinearity with 
Table 2 Coefficients for the full mediation model of sex differences in spatial navigation

\begin{tabular}{lllll}
\hline & Unstandardized coefficients & Std. Error & $p$ value & $R^{2}$ \\
\hline Latent exploration variable loadings & & & & \\
$\quad$ Pausing & 1.000 & 0 & 0 & .818 \\
$\quad$ Revisiting & 0.482 & 0.067 & $<.001$ \\
$\quad$ Directional Persistence & -0.063 & 0.009 & .675 \\
Navigational success on & & & $<.001$ & .299 \\
$\quad$ Latent exploration variable & -.421 & .109 & .268 \\
$\quad$ Gender & .321 & .294 & .001 \\
$\quad$ SBSOD & 2.368 & 1.984 & .401 & .001 \\
Latent exploration variable on & & & .499 & \\
$\quad$ Gender & -.434 & .101 & $<.001$ \\
\hline
\end{tabular}

the wayfinding behaviors. Also, it is possible that the SBSOD would predict real-world wayfinding in a nonmilitary population. More work is needed to test these alternatives.

\section{Limitations}

This study is one of few large-scale wayfinding tasks conducted in a real environment, and as such the measures created for this study have not been extensively validated. Further, this work was done with a self-selected sample, and these results may not be generalizable to the lay population. More work must be done to both determine whether these effects hold in similar tasks and establish if the wayfinding behaviors tested herein exist in other goal-oriented navigation tasks.

It has been proposed that the difference between mapbased tasks and use of ecological environments is the determining factor as to whether gender differences are found. Specifically, men perform better on map-based tasks, yet this difference is not always reflected in ecological navigation tasks (Galea \& Kimura, 1993; Halpern \& Wright, 1996; Rossano \& Moak, 1998). Because our navigational task required the use of both a map and compass, some unreported difference in the comfort level with the use of these tools might contribute to the observed gender difference in performance. However, we did include comfort with navigational

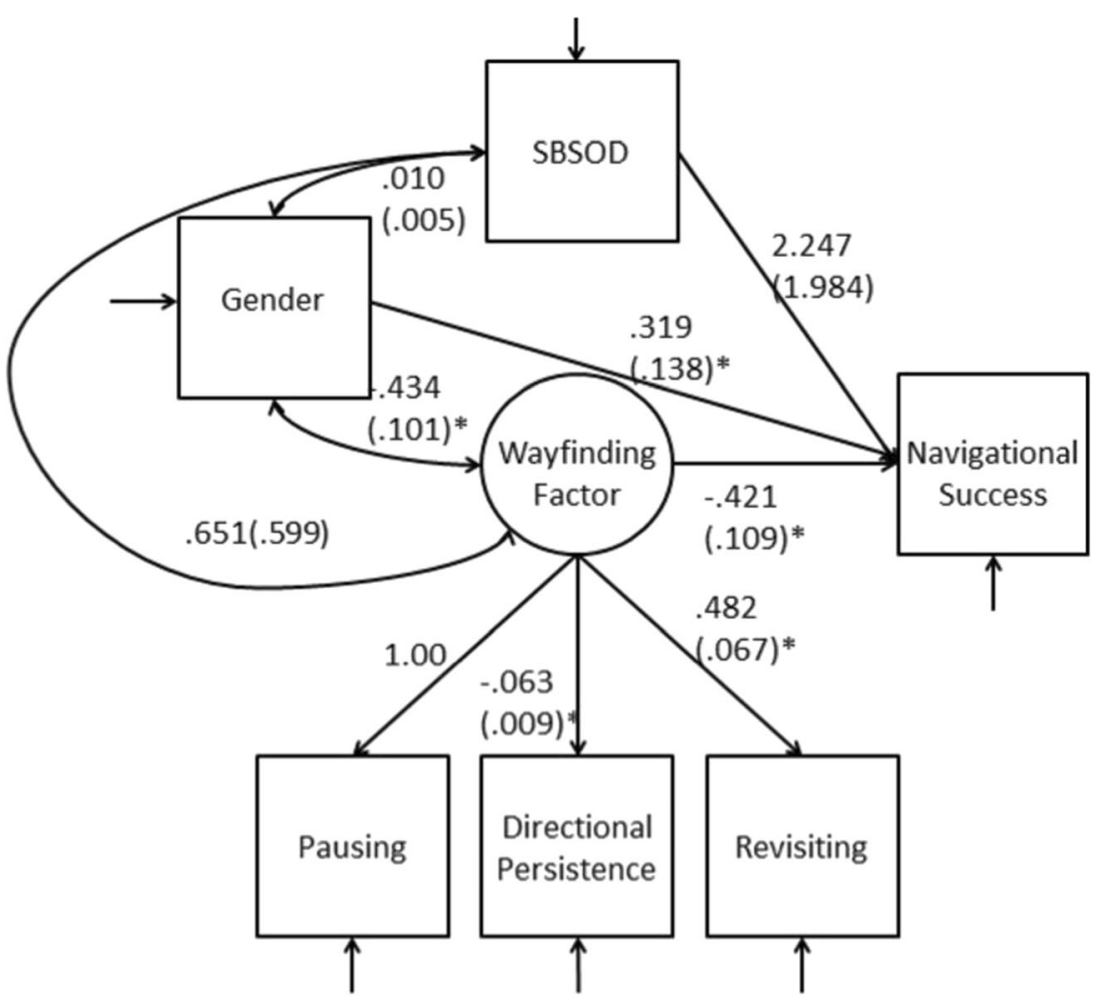

Fig. 2 Nested nonmediation model. Standard errors are in parenthesis. * = significance at the .05 level 
tools as a covariate, to attempt to control for this potential confound. Further, all participants demonstrated proficiency with the map and compass during training.

\section{Conclusions}

This is one of few studies that investigate how wayfinding contribute to navigational performance on a large-scale, realworld navigation task. Further, this study attempted to answer whether men and women move through the environments differently and if those differences in wayfinding behaviors are related to the ability to locate a target. This study contributes to validating novel characterizations of how people move through real-world environments when searching for targets. It also demonstrates that people producing more directional persistence, and less pausing and revisiting, successfully located more targets (flags). Cadets with higher degrees of directional persistence and lower degrees of revisiting and pausing behaviors located more correct flags within the larger cluster of flags. As predicted by our hypotheses, men produced more directional persistence, less pausing, and less revisiting compared with women, which in turn related to more navigational success. These findings were consistent with wayfinding behaviors mediating the relationship between gender and navigational performance. Thus, gender is associated with the type of wayfinding behaviors someone is likely to produce, and how people move through the environment is related to their ability to locate targets.

Future work may seek to connect commonly used measures in spatial cognition and navigation that show gender differences with the wayfinding behaviors, since gender may be acting as a proxy for another variable that affects wayfinding and shows larger gender differences. This could further disentangle what causes the differences in wayfinding behaviors. For example, individual differences in spatial anxiety or caution might be tied to the likelihood of participants pausing or revisiting more. Importantly, attempting to increase directional persistence and decrease pausing or revisiting behavior is not likely to level individual navigational differences. Instead, we expect that targeting potential underlying causes of individual differences in wayfinding behaviors (for example, reducing spatial anxiety that theoretically could lead to caution when navigating) rather than manipulating the wayfinding behaviors may better improve navigational success. These causes must be identified, and gender may just be a proxy or an earlier link in the meditational chain for them. These variables could be environmental, biological, or social. For example, it could be circulating hormone load, genetics, or something society socializes differently by gender (i.e., how children are allowed to play and interact with the world from a young age), which leads to differences in wayfinding behaviors. Finally, confirming that similar patterns are present in different navigational tasks, such as tasks using technological navigational aids but not a map and compass, or non-nature-based navigational tasks, is necessary. This study suggests that the proposed measures of wayfinding behaviors may be the start of a functional taxonomy of how people move through space.

Acknowledgements This work was partially supported by the National Science Foundation Grant No. 1329091, and the Office of Naval Research Grant N00014-18-1-2964.

\section{References}

Boone, A. P., Gong, X., \& Hegarty, M. (2018). Sex differences in navigation strategy and efficiency. Memory \& Cognition, 1-14. Advance online publication. https://doi.org/10.3758/s13421-018-0811-y

Castelli, L., Latini-Corazzini, L., \& Geminiani, G. C. (2008). Spatial navigation in large-scale virtual environments: Gender differences in survey tasks. Computers in Human Behavior, 24(4), 1643-1667. https://doi.org/10.1016/j.chb.2007.06.005

Coluccia, E., \& Louse, G. (2004). Gender differences in spatial orientation: A review. Journal of Environmental Psychology, 24(3), 329340. https://doi.org/10.1016/j.jenvp.2004.08.006

Fields, A. W., \& Shelton, A. L. (2006). Individual skill differences and large-scale environmental learning. Journal of Experimental Psychology: Learning, Memory, and Cognition, 32(3), 506-515. https://doi.org/10.1037/0278-7393.32.3.506

Gagnon, K. T., Cashdan, E. A., Stefanucci, J. K., \& Creem-Regehr, S. H. (2016). Sex differences in exploration behavior and the relationship to harm avoidance. Human Nature, 27(1), 82-97. https://doi.org/10. 1007/s12110-015-9248-1

Gagnon, K. T., Thomas, B. J., Munion, A., Creem-Regehr, S. H., Cashdan, E. A., \& Stefanucci, J. K. (2018). Not all those who wander are lost: Spatial exploration patterns and their relationship to gender and spatial memory. Cognition, 180, 108-117. https://doi. org/10.1016/j.cognition.2018.06.020

Galea, L. A., \& Kimura, D. (1993). Sex differences in route-learning. Personality and Individual Differences, 14(1), 53-65. https://doi. org/10.1016/0191-8869(93)90174-2

Graham, J. W. (2009). Missing data analysis: Making it work in the real world. Annual Review of Psychology, 60, 549-576. https://doi.org/ 10.1146/annurev.psych.58.110405.085530

Halpern, D. F., \& Wright, T. M. (1996). A process-oriented model of cognitive sex differences. Learning and Individual Differences, 8(1), 3-24. https://doi.org/10.1016/S1041-6080(96)90003-5

Hegarty, M., Montello, D. R., Richardson, A. E., Ishikawa, T., Lovelace, K. (2006). Spatial abilities at different scales: Individual differences in aptitude-test performance and spatial-layout learning. Intelligence, 34(2), 151-176.

Hegarty, M., Richardson, A. E., Montello, D. R., Lovelace, K., \& Subbiah, I. (2002). Development of a self-report measure of environmental spatial ability. Intelligence, 30(5), 425-447. https://doi. org/10.1016/S0160-2896(02)00116-2

Hu, L. T., \& Bentler, P. M. (1999). Cutoff criteria for fit indexes in covariance structure analysis: Conventional criteria versus new alternatives. Structural Equation Modeling: A Multidisciplinary Journal, 6(1), 1-55. https://doi.org/10.1080/10705519909540118

Hund, A. M., \& Minarik, J. L. (2006). Getting from here to there: Spatial anxiety, wayfinding strategies, direction type, and wayfinding efficiency. Spatial Cognition and Computation, 6(3), 179-201. https:// doi.org/10.1207/s15427633scc0603_1

Ishikawa, T., \& Montello, D. R. (2006). Spatial knowledge acquisition from direct experience in the environment: Individual differences in 
the development of metric knowledge and the integration of separately learned places. Cognitive Psychology, 52(2), 93-129. https:// doi.org/10.1016/j.cogpsych.2005.08.003

Kenny, D. A. (2008). Reflections on mediation. Organizational Research Methods, 11(2), 353-358. https://doi.org/10.1177/ 1094428107308978

Malinowski, J. C. (2001). Mental rotation and real-world wayfinding. Perceptual and Motor Skills, 92(1), 19-30. https://doi.org/10.2466/ pms.2001.92.1.19

Malinowski, J. C., \& Gillespie, W. T. (2001). Individual differences in performance on a large-scale, real-world wayfinding task. Journal of Environmental Psychology, 21(1), 73-82. https://doi.org/10.1006/ jevp. 2000.0183

Montello, D. R., Lovelace, K. L., Golledge, R. G., \& Self, C. M. (1999). Sex-related differences and similarities in geographic and environmental spatial abilities. Annals of the Association of American Geographers, 89(3), 515-534. https://doi.org/10.1111/0004-5608. 00160

Ploran, E. J., Rovira, E., Thompson, J. C., \& Parasuraman, R. (2015). Underlying spatial skills to support navigation through large, unconstrained environments. Applied Cognitive Psychology, 29(4), 608613. https://doi.org/10.1002/acp.3135

Rossano, M. J., \& Moak, J. (1998). Spatial representations acquired from computer models: Cognitive load, orientation specificity and the acquisition of survey knowledge. British Journal of Psychology, 89(3), 481-497. https://doi.org/10.1111/j.2044-8295.1998.tb02698.x
Rovira, E., Mackie, R. S., Clark, N., Squire, P. N., Hendricks, M. D., Pulido, A. M., \& Greenwood, P. M. (2016). A role for attention during wilderness navigation: Comparing effects of BDNF, KIBRA, and CHRNA4. Neuropsychology, 30(6), 709-719. https:// doi.org/10.1037/neu0000277

Sandstrom, N. J., Kaufman, J., \& Huettel, S. A. (1998). Males and females use different distal cues in a virtual environment navigation task. Cognitive Brain Research, 6(4), 351-360. https://doi.org/10. 1016/S0926-6410(98)00002-0

Silverman, I., Choi, J., Mackewn, A., Fisher, M., Moro, J., \& Olshansky, E. (2000). Evolved mechanisms underlying wayfinding: Further studies on the hunter-gatherer theory of spatial sex differences. Evolution and Human Behavior, 21, 201-213. https://doi.org/10. 1016/S1090-5138(00)00036-2

Turchin, P. (1998). Quantitative analysis of movement: Measuring and modeling population redistribution of plants and animals. Sunderland: Sinauer Associates.

Weisberg, S. M., Schinazi, V. R., Newcombe, N. S., Shipley, T. F., \& Epstein, R. A. (2014). Variations in cognitive maps: Understanding individual differences in navigation. Journal of Experimental Psychology: Learning, Memory, and Cognition, 40(3), 669-682. https://doi.org/10.1037/a0035261

Publisher's note Springer Nature remains neutral with regard to jurisdictional claims in published maps and institutional affiliations. 